\title{
Shape transformers for material and shape selection of lightweight beams
}

\author{
Damiano Pasini * \\ McGill University, Department of Mechanical Engineering, 817 Sherbrooke Street West, Montreal, Canada H3A 2 K6
}

Received 11 November 2005; accepted 25 May 2006

Available online 13 July 2006

\begin{abstract}
This paper presents a method for selecting materials, cross-section shapes, and combinations thereof. The novelty of the method is the definition of shape transformers. These parameters are dimensionless measures of the geometric quantities of a cross-section. They describe the shape properties regardless of size, and are invariant to any scaling imposed on the size of a beam cross-section in bending. Shape transformers are valuable to model the equations of mechanics and to develop selection charts for optimum design. The rationale of the approach is that the fundamental equations of continuum mechanics can be expressed by a product of four factors: the functional requirements, the material properties, the shape transformers, and the geometric quantities of a rectangle as defined by its cross-section size. This permits general expressions of performance indices to be derived for any scaling transformation. Indices for selecting materials and cross-sectional shapes that minimize the mass of beams are given for stiffness design. The last part of the paper illustrates how shape transformers facilitate a graphical exploration of performance data. The whole range of cross-sectional shapes can be visualized at a glance for each material. Lines of iso-performance enable efficiency comparison of materials and/or shapes for a given cross-section scaling. Shape transformers assist design choices and give insight into optimum selection.
\end{abstract}

(c) 2006 Elsevier Ltd. All rights reserved.

Keywords: Material selection charts $(\mathrm{H})$; Weighting and ranking factors $(\mathrm{H})$; Beams $(\mathrm{N})$

\section{Introduction}

Making products that are cheap and have high technical performance is a common demand of the industrial market. The task can be challenging for engineers because they have to explore several solutions in a limited time and avoid choices that result in a poor product. The burden of the struggle can be alleviated if they adopt a strategy that assists them in making good decisions. The method should be effective in providing a large number of alternatives, a prompt ranking of their performance, and a guide for the selection of the best solution.

In the design of light components, materials and crosssection shapes govern structural efficiency. They are crucial

\footnotetext{
* Tel.: +1 514398 6295; fax: +1 5143987365 .

E-mail address: damiano.pasini@mcgill.ca.
}

variables that have a large impact on performance. For this reason, it is not surprising that they were the focus of extensive research in the past [1-5]. Minimum weight criteria were developed to compare the lightweight potential of materials and cross-sectional shapes. The criteria were often used in combination with charts that plot how the efficiency of a solution changes with the design variables $[1-3,5]$. The charts offer a valuable means for making early design choices. Ashby [5,6], for example, has demonstrated that charts and indices of selection can be successfully combined to support the selection of the best material for an application, and also to design new materials [7].

This paper presents a novel strategy to support the selection of materials, cross-sectional shapes and their co-selection. The framework of the method is underpinned by the concept of shape transformers $[8,9]$. Shape transformers are dimensionless parameters defined to measure geometric quantities of a cross-section regardless of size. In bending 
stiffness design, shape transformers describe shape properties that are invariant to any scaling of a cross-section. Their definition generally permits to decouple the shape properties from the cross-section size in an equation of mechanics. The latter can be expressed by a product of four terms: the design specifications, the material attributes, the shape transformers, and the geometric quantities of the rectangular envelope, whose dimensions are the cross-section sizes. The second part of the paper shows an application of the rationale for deriving performance indices of beams in stiffness design. The indices allow an independent choice of materials and shapes for any scaling transformation. The final part of the paper illustrates that shape transformers enable a simultaneous plot of performance for different combinations of materials and shape properties. These charts facilitate the comparison among various design options, and provide insight into the variables that govern performance.

\section{The basics of the methodology}

This section presents the fundamentals of the methodology and is divided in four parts. The first introduces a concept for describing the transversal geometry of an element in terms of shape and envelope. Then, the concept is used to define classes of shapes and scalar operators of design: the shape transformers and the envelope multiplicators. This is followed by the rationale of the method.

\subsection{Shape and envelope of a cross-section}

Engineering design often requires decisions about the geometry of structural components. When a material is shaped into a structural element, its geometry can be described by a variety of geometric quantities, such as the area and perimeter of its cross-section, as well as its volume.

The idea of measuring the geometry of a cross-section with two separate entities is now introduced. The first entity is related to the cross-section sizes defining a rectangular envelope, $D$. The other describes the shape, $S$, of the figure enclosed in the envelope, $D$. Fig. 1 illustrates $S$ and $D$ for three generic cross-sections. The shape, $S$, changes in all

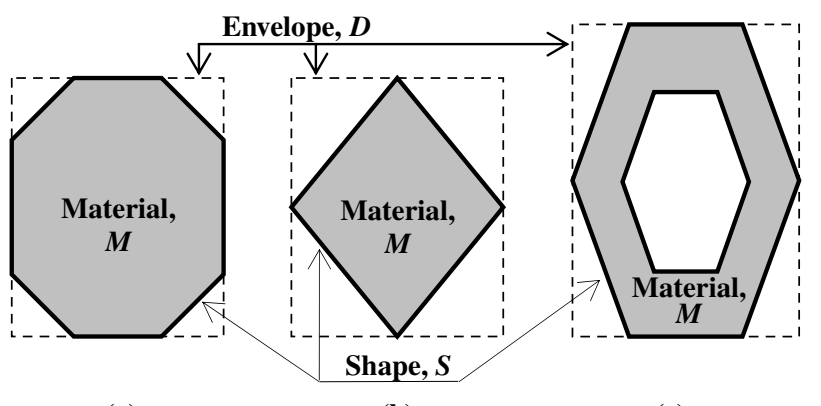

(a)

(b)

(c)

Fig. 1. The material, $M$, the shape, $S$, and the envelope, $D$, of common cross-sections. examples, while the envelope, $D$, is the same for the crosssections shown in Figs. 1(a) and (b).

A cross-section is therefore conceived as a material, $M$, moulded into a shape, $S$, that fits within a rectangular envelope, $D$. The design parameters $S$ and $D$ are defined in the following sections.

\subsection{Shape transformers defining shape classes}

Specifying shape properties separable from the properties of the envelope leads to the idea of defining $S$ as invariant to $D$. This concept is formulated in terms of shape transformers, $S$. Shape transformers are measures of the geometric quantities of an object. They can be defined for the area, the volume, and others quantities of an object, regardless of size [8]. $S$ is governed by the shape of the object but does not vary with size changes.

Definition: If $G$ is the measure of a geometric quantity, and $G_{D}$ measures the geometric quantity of its envelope, then a shape transformer is defined as:

$S=\frac{G}{G_{D}}$

Consider, for example, two geometric measures of a cross-section, such as the area, $A$, and the second moment of area about the bending axis, $I$. Table 1 lists $A$ and $I$ for some common cross-sections symmetric about vertical and horizontal envelope mid-planes. If $A_{D}$ and $I_{D}$ are the respective quantities of the envelope, then applying Eq. (1) gives the shape transformers for the area, $\psi_{A}$, and for the second moment of area, $\psi_{I}$ :

$\left\{\begin{array}{l}\psi_{A}=\frac{A}{A_{D}} \\ \psi_{I}=\frac{I}{I_{D}}\end{array}\right.$

Shape transformers have been formulated to specify classes of shapes similar to material classification. Rectangles, ellipses, and diamonds, for example, define different shape classes $[7,8]$ as metals, ceramics and composites

Table 1

Area, $A$, and second moment of area about $x x, I_{x x}$, of common crosssections

\begin{tabular}{lll}
\hline Cross-section & $A$ & $I_{x x}$ \\
\hline & & \\
\hline
\end{tabular}


Table 2

Shape transformers for the area and second moment of area of cross-sections with $c=b / B$ and $d=h / H$

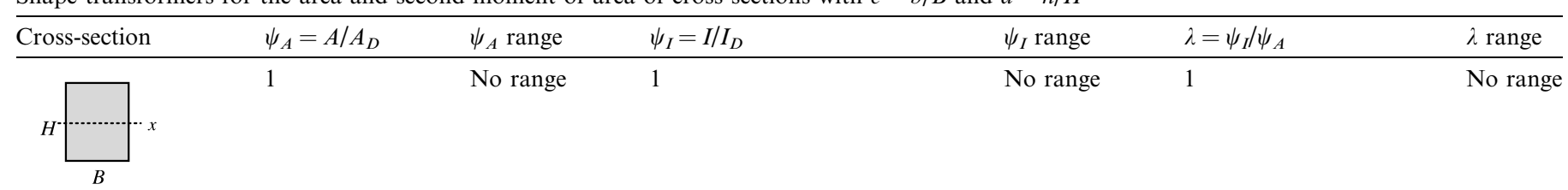

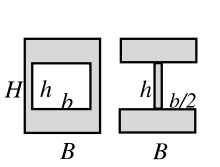
$1-c d$
$0-1$
$1-c d^{3}$ for $c \neq d \neq 1$
$0-1$
$\frac{1-c d^{3}}{1-c d}$
$1-3$

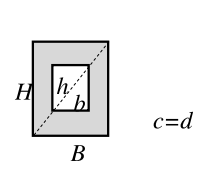
$1-d^{2}$
$0-1$
$2 \psi_{A}-\psi_{A}^{2}$
$0-1$
$2-\psi_{A}$

$1-2$

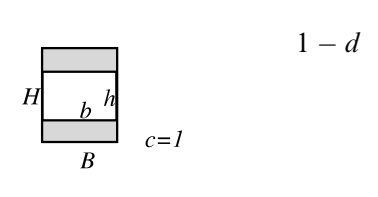

$0-1 \quad \psi_{A}^{3}-3 \psi_{A}^{2}+3 \psi_{A}$

$0-1$

$\psi_{A}^{2}-3 \psi_{A}+3$

$1-3$

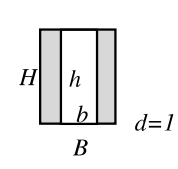
$1-c$
$0-1$
$\psi_{A}$

$0-1$

1

No range

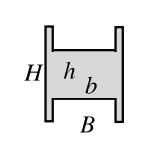

$$
1-c(1-d)
$$

$0-1$

$1-c\left(1-d^{3}\right)$ for $c \neq d \neq 1$

$0-1$

$\frac{1-c\left(1-d^{3}\right)}{1-c(1-d)}$

$0-1$

$\psi_{A}^{3}$ for $c=1$

$\psi_{A}^{2}$ for $c=1$

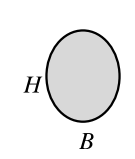

$\frac{\pi}{4}$

No range $\quad \frac{3 \pi}{16}$

No range

$\frac{3}{4}$

No range

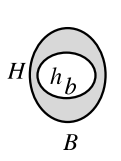

$$
\frac{\pi}{4}(1-c d)
$$

$0-\pi / 4$

$\frac{3 \pi}{16}\left(1-c d^{3}\right)$

$0-3 \pi / 16$

$\frac{3}{4}\left(\frac{1-c d^{3}}{1-c d}\right)$

3/4-9/4

$\underbrace{}_{B}(b)$

$$
\frac{\pi}{4}\left(1-d^{2}\right)
$$

$0-\pi / 4$

$\frac{3}{2} \psi_{A}\left(1-\frac{2}{\pi} \psi_{A}\right)$

$0-3 \pi / 16$

$\frac{3}{2}\left(1-\frac{2}{\pi} \psi_{A}\right)$

$3 / 4-3 / 2$

$\overbrace{B}^{h_{B}}$

$\frac{\pi}{4}(1-d)$

$0-\pi / 4$

$\frac{3}{4} \psi_{A}\left(3-\frac{12}{\pi} \psi_{A}+\frac{16}{\pi^{2}} \psi_{A}^{2}\right)$

$0-3 \pi / 16$

$\frac{3}{4}\left(3-\frac{12}{\pi} \psi_{A}+\frac{16}{\pi^{2}} \psi_{A}^{2}\right)$

3/4-9/4

$H(\underbrace{}_{B} \begin{array}{l}h \\ b\end{array})_{d=1}$

$\frac{\pi}{4}(1-c)$

$0-\pi / 4$

$\frac{3}{4} \psi_{A}$

$0-3 \pi / 16$

$\frac{3}{4}$

No range 
describe classes of materials. Each shape class contains a solid cross-section and all the hollow cross-sections derived by the solid one. This means that a hollow cross-section belongs to a particular class only if the shape of the removed internal material is the same of that of its solid cross-section. For example, a hollow section falls into the class of the ellipses if the removed material has an elliptical shape; while a hollow rectangular cross-section is included in the rectangles class because the removed internal layer is a rectangle. Table 2 reports the shape transformers for the ellipses and rectangles classes. The main dimensions of the cross-sections, $B$ and $H$, describe the envelope, $D$. Since $S$ are defined regardless of size, circular and square cross-sections fit into the classes of the ellipses and rectangles, respectively.

The special case where the shape completely fills the envelope is a rectangle or square section, where $G=G_{D}$ and $S$ is unity, such that:

$$
\left\{\begin{array}{l}
A=A_{D} \rightarrow \psi_{A}=1 \\
I=I_{D} \rightarrow \psi_{I}=1
\end{array}\right.
$$

For any other shape, $S$ is less than one (Table 2). Solid cross-sections, such as ellipses, hexagons, polygonal figures, and any other shapes, have definite $S$. Hollow shapes are derived by removing material layer from their solid shapes and, therefore, $S$ ranges with the location of the material within the envelope. Domains of the shape transformers are between zero, i.e. empty envelope, and the values of the respective solid section. These are upper limit, representing cross-sections, where the material completely fills the solid shapes. When these transformers (Table 2) are used to characterize the efficiency of cross-sections, then the ranking of a wide range of shapes can be visualized in a single design chart for different shape classes. This will be shown in Section 4.

\subsection{Envelope multiplicators}

While $S$ is fundamental for comparing shape properties among cross-sections, differences in cross-section sizes (Figs. 1(b) and (c)) can be assessed in terms of envelope multiplicators. These parameters describe size changes between cross-sections, i.e. they express the relative scaling of two envelopes.

To deal with the relative scaling of cross-sections, we chose a reference cross-section: a rectangle. This is described by width, $B_{\mathrm{o}}$, height, $H_{\mathrm{o}}$, geometric quantities, $G_{\mathrm{o}}$, such as $A_{\mathrm{o}}$ for area and $I_{\mathrm{o}}$ for second moment of area, and shape transformers $\psi_{A}=\psi_{I}=1$ because $G_{\mathrm{o}}=G_{D}$.

Now consider a generic cross-section with dimensions $B$ and $H$. The changes in sizes relative to the reference can be conveniently described in bending stiffness design by the following multiplicators, $u$ and $v$ :

$$
\left\{\begin{array}{l}
u=\frac{B}{B_{\mathrm{o}}}=\frac{b}{b_{\mathrm{o}}} \\
v=\frac{H}{H_{\mathrm{o}}}=\frac{h}{h_{\mathrm{o}}}
\end{array}\right.
$$

where $b$ and $b_{\mathrm{o}}, h$ and $h_{\mathrm{o}}$ are the dimensions of the internal layer for hollow or open cross-sections (Table 2).

Three examples of scaling an elliptical cross-section are shown in Fig. 2. While $S$ is invariant to scaling transformations, $u$ and $v$ assume different values. In bending stiffness design, there are three directions of scaling where $u$ and/ or $v$ have special values: $v=1$ for horizontal, $u=1$ for vertical, and $u=v$ for proportional scaling. Reasons for imposing a scaling to a cross-section in certain directions include geometric constraints, size and shape availability, and type of applied loading $[8,10,11]$.

\subsection{The fundamental rationale}

Defining shape properties that are invariant to scaling can be advantageous with writing the equations of continuous mechanics, such as those describing failure mechanisms. An equation of mechanics, E.M., can often be expressed in terms of material properties, $M$, geometry, $G$, and problem specifications, $F$, in the form:

$$
\text { E.M. }=F \times M \times G
$$

If the shape transformer definition (1) is rearranged and used to substitute $G$ in Eq. (4), then $S$ and $G_{D}$ can be decoupled and the E.M. can be written as:

E.M. $=F \times M \times S \times G_{D}$

where $F$ groups the design specifications; $M$ are the material properties, such as the density, $\rho$, and the Young's Modulus, $E ; S$ are the shape transformers of the cross-section, such as $\psi_{A}$ and $\psi_{I} ; G_{D}$ are the geometric quantities of the envelope, $D$, that are functions of the cross-section sizes, such as $A_{D}$ and $I_{D}$.

Eq. (5) represents the underlying principle of the shape transformers method. An equation of mechanics can be expressed by a product of $F, M, S$, and $D$ [8]. As will be shown in Section 3, this feature is valuable in gaining insight about the role that the variables $M, S$, and $D$ play in a failure mechanism and in characterizing the performance.

The rationale of the shape transformers can be applied to a number of design cases. As an example, the following section presents performance indices for the design of

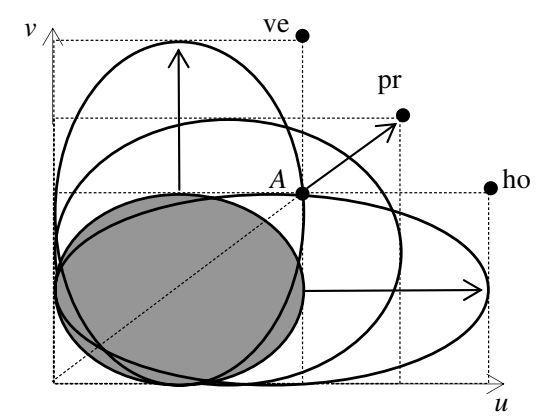

Fig. 2. Examples of scaling a section $A$. For horizontal (ho) scaling $v=1$, for proportional (pr) scaling $v=u$, for vertical (ve) scaling $u=1$. 
beams, while Section 4 illustrates the efficacy of the rationale with selection charts.

\section{Lightweight design of stiff beams in bending}

\subsection{Modelling with the shape transformers}

This section considers the design of light weight beams that must not deflect more than $\delta$ under a load $P$ (Fig. 3). In this scenario, the stiffness requirement of the beam is $P / \delta$ and its mass is to be as low as possible. The equations of stiffness and mass are those relevant here.

Stiffness. The stiffness of a beam of given length, $L$, and boundary conditions, $c_{1}$, is described by the equation:

$k=\frac{c_{1}}{L^{3}} E I$

where $I$ is the second moment of area and $E$ is the Young's Modulus.

Eq. (6) is written in the classic form as in (4). If the shape Transformer $\psi_{I}$ is rearranged to substitute $I$ in Eq. (6), the beam stiffness takes the form of Eq. (5):

$k=\frac{c_{1}}{L^{3}} \times E \times \psi_{I} \times \frac{B H^{3}}{12}$

Mass. Similarly, if the shape transformer $\psi_{A}$ is rearranged to substitute $A$ in the mass expression $m=\rho A L$, the latter takes the form of Eq. (5) as well:

$m=L \times \rho \times \psi_{A} \times B H$

Now rearranging expressions (7) and (8) so that the design variables are isolated to the right-hand side of the equations gives:

$m=\rho \times \psi_{A} \times A_{D}$

$F^{\prime}=E \times \psi_{I} \times I_{D}$

where $m$ is the mass per unit length of the beam and $F^{\prime}=k L^{3} / c_{1}$ groups the design specifications.

This rearrangement is helpful in visualizing the variables and the performance of a cross-section into design charts. This will be illustrated in Section 4 .

\subsection{Indices of selection}

The methodology is now applied to derive indices that maximize the performance of beams in two design scenarios.

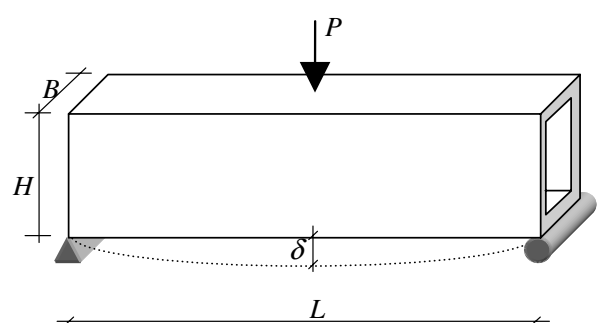

Fig. 3. Simply-supported beam under a central load $P$.
The first case (Section 3.2.1) examines beams of equal sizes such that the envelope is constrained in both width and height. The second case (Section 3.2.2) examines the condition where the design allows the cross-sections to be scaled.

\subsubsection{Performance index for non-scaled cross-sections}

When cross-sections have the same sizes, $u=v=1$, i.e. there is no relative scaling. The geometric quantities, $G_{D}$, of the envelope do not vary and the variables are $M$ and $S$. We derive a performance index to select the beam that best minimizes mass. The higher the performance index, the lighter the cross-section.

Consider now the ratio of the functional requirement, expression (10), to the objective function, expression (9):

$\frac{F^{\prime}}{m}=\frac{E}{\rho} \frac{\psi_{I}}{\psi_{A}} \frac{I_{D}}{A_{D}}$

If the constants are grouped into the design requirement $F^{\prime \prime}=P L^{3} A_{D} / \delta c_{1} I_{D}$ and expression (11) is rearranged to yield the inverse of mass, $1 / m$, then:

$\frac{1}{m}=\underbrace{\frac{1}{F^{\prime \prime}}}_{\text {const }} \underbrace{\frac{E}{\rho} \frac{\psi_{I}}{\psi_{A}}}_{\text {var }}$

Expression (12) can be used to find performance indices for three types of selection, as will be demonstrated below.

$M$ and $S$ co-selection. Since $F^{\prime \prime}$ collects the specifications of the design, and $M$ and $S$ are the variables, the selection index, $p$, which governs the performance is a combination of the following properties:

$p=\frac{E}{\rho} \frac{\psi_{I}}{\psi_{A}}$

The optimum cross-section will have a combination of material attributes and shape transformers that best maximize the performance, $p$.

$M$ selection. If the design requires only the choice of the material because the shape, $S$, is already prescribed, then the index for material selection, $p_{m}$, is

$p_{m}=\frac{E}{\rho}$

$S$ selection. If $M$ is given, shape selection is ruled by the index for shape selection, $p_{s}$ :

$p_{s}=\frac{\psi_{I}}{\psi_{A}}$

Expression (15) has been recently defined as the envelope efficiency parameter, $\lambda[8,9]$. Expressions, as well as ranges of $\lambda$, have been formulated for different shape classes, as reported in Table 2. Note that these ranges are theoretical because there are manufacturing constraints and local instability that limit the shaping of a material into a thin wall. These empirical limits have been examined for practical steel cross-sections [8,9]. Failure due to shear is also an issue to be considered. More details on the meaning of $\lambda$ will be given in Section 4 with selection charts. 


\subsubsection{Performance index for scaled cross-sections}

In contrast to the previous section, the selection of beams having cross-sections of various sizes is now considered. Allowing envelope variation implies that the candidate cross-sections are relatively scaled. Examples of scaling transformations are shown in Fig. 2. Cross-section $A$ can be scaled in either a horizontal (ho), vertical (ve), or proportional (pr) direction. In all other cases, the scaling is arbitrary since $u \neq v \neq 1$.

Expression (13), $p=\lambda E / \rho$, is not generally appropriate when the beams are relatively scaled. Performance indices for different scaling conditions are given here.

\subsubsection{Selection for arbitrary scaling. $M$ and $S$ co-selection.}

The general solution of the performance index, $\bar{p}$, for an arbitrary scaling of the cross-section in bending stiffness design $[8,10]$ is

$\bar{p}=\frac{\left(E \psi_{I}\right)^{q}}{\rho \psi_{A}}$

where $q=\ln u v / \ln u v^{3}$ is a scaling function of the envelope multiplicators, $u$ and $v$. The derivation of $\bar{p}$ is not reported here due to space constraint. The reader is referred to $[8,10]$.

$M$ selection. If the material is the design variable for arbitrarily scaled cross-sections that have a given shape, $S$, then the index for material selection, $\bar{p}_{m},[8,11]$ is

$\bar{p}_{m}=\frac{E^{q}}{\rho}$

$S$ selection. If $M$ is prescribed, shape selection for scaled cross-sections is ruled by the index for shape selection, $\bar{p}_{s}$ $[8,10]$ :

$\bar{p}_{s}=\frac{\psi_{I}^{q}}{\psi_{A}}$

The indices given by expressions (16)-(18) permit the selection of cross-sections that can be scaled in any direction, including an inclined angle to the horizontal [8].

3.2.2.2. Selection for prescribed scaling. When the scaling transformation is decided a priori or imposed by the design, such as with tightly constrained structures, the exponent $q$ becomes known and the indices are solely functions of $M$ and/or $S$. The effect of an early setting of scaling on the performance is shown in Table 3, where the indices are given for horizontal, vertical, and proportional scaling.

Table 3

Performance indices for three scaling transformations

\begin{tabular}{llll}
\hline Scaling & $\begin{array}{l}\text { Material } \\
\text { selection }\end{array}$ & $\begin{array}{l}\text { Shape } \\
\text { selection }\end{array}$ & $\begin{array}{l}\text { Material and } \\
\text { shape selection }\end{array}$ \\
\hline Horizontal, $v=1$ & $\frac{E}{\rho}$ & $\frac{\psi_{I}}{\psi_{A}}$ & $\frac{E}{\rho} \lambda$ \\
Vertical, $u=1$ & $\frac{E^{1 / 3}}{\rho}$ & $\frac{\psi_{I}^{1 / 3}}{\psi_{A}}$ & $\frac{\left(E \psi_{I}\right)^{1 / 3}}{\rho \psi_{A}}$ \\
Proportional, $u=v$ & $\frac{E^{1 / 2}}{\rho}$ & $\frac{\psi_{I}^{1 / 2}}{\psi_{A}}$ & $\frac{\left(E \psi_{I}\right)^{1 / 2}}{\rho \psi_{A}}$ \\
\hline
\end{tabular}

Results in Table 3 have been derived using expressions (16)-(18). When inserted in the expression $q=\ln u v / \ln u v^{3}$, horizontal scaling yields $q=1$, vertical scaling yields $q=1 / 3$, and proportional scaling yields $q=1 / 2$. These indices are now used for graphical selection purposes.

\section{Shape transformers with selection charts}

Performance indices for material selection (first column of Table 3) have been successfully applied with material charts by Ashby [5-7]. This section presents a graphical approach to visualize how shape transformers can improve the selection of materials and shapes.

\subsection{Key reading of the selection charts}

Using the shape transformers, a selection chart displays one or a combination of properties of the objective function, $\rho \times \psi_{A} \times A_{D}$ (expression (9)), versus one or a combination of properties of the design requirements, $E \times \psi_{I} \times I_{D}$ (expression (10)). In the design of a stiff light beam, the properties of mass that are variable are reported on the abscissa, while the selectable attributes that describe the functional requirements are reported on the ordinate. This means that the location of a cross-section $C$ on a chart is identified by a point $C$ with co-ordinates:

$C(\underbrace{\rho \times \psi_{A} \times A_{D}}_{m}, \underbrace{E \times \psi_{I} \times I_{D}}_{F^{\prime}})$

The following examines the graphical selection of the cross-section variables by using the indices given in Section 3.2.1 for non-scaled cross-sections and Section 3.2.2 for scaled cross-sections.

\subsection{Shape selection charts for non-scaled cross-sections}

This design task requires the choice of the best shape for a cross-section with prescribed material and sizes. The appropriate index is given by expression (15), i.e. $p_{s}=\psi_{I} /$ $\psi_{A}$. Since $M$ and $D$ are constant, the position of a cross-section on a chart is given by the co-ordinates:

$C\left(\frac{m}{\rho \times A_{D}}=\psi_{A}, \frac{F^{\prime}}{E \times I_{D}}=\psi_{I}\right)$

The shape transformers $\psi_{A}$ and $\psi_{I}$, given in Table 2, are used to plot the curves in Fig. 4 for rectangles and ellipses. This chart, called the envelope efficiency map, has been recently developed for a different shape classes $[8,9]$.

A description of the chart is now briefed. All the crosssectional shapes that partially fill the envelope, $B \times H$, occupy an area bordered by limit curves $1 \mathrm{v}$ and $2 \mathrm{v}$. The curves represent performance of the rectangles shape class when the material fraction varies within an envelope of any size. Outside this area, no cross-sectional shape exists. Limit curve $1 \mathrm{v}$ represents conditions where the material 


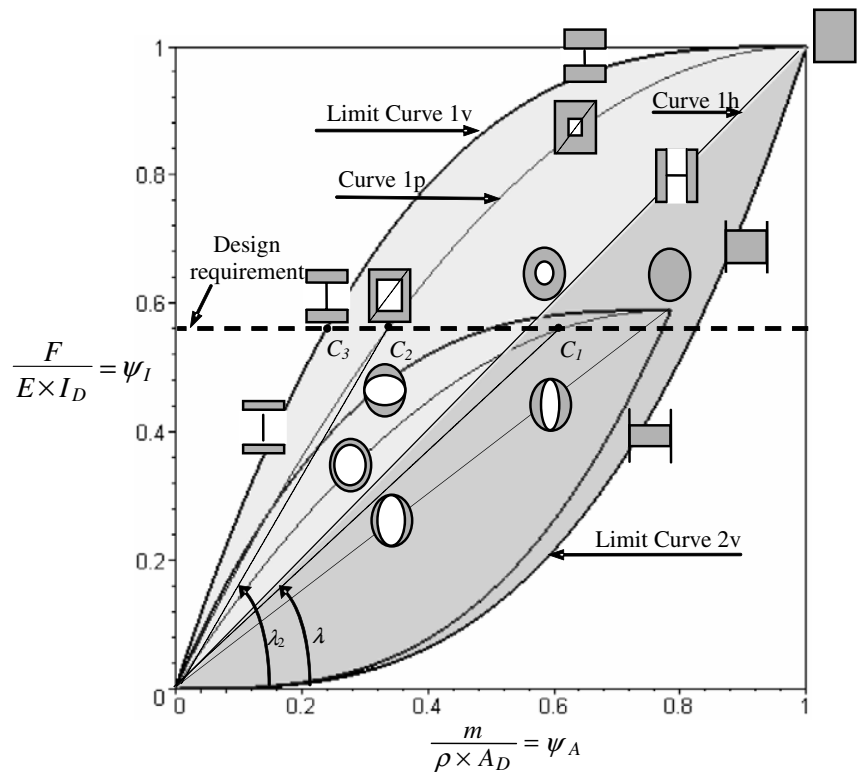

Fig. 4. Selection chart for shapes not scaled. Legend: light grey for efficient region, dark grey for inefficient region.

layer placed on the upper and lower surfaces of the crosssection is scaled vertically. Limit curve $2 \mathrm{v}$ describes an idealised $\mathrm{H}$ cross-section where the material layer is placed in the middle and is vertically scaled. Curve $1 \mathrm{p}$ illustrates a hollow beam where the material on the outer surfaces is proportionally scaled. Idealised $\mathrm{H}$ cross-sections with horizontally scaled layers are represented by curve $1 \mathrm{~h}$. Cross-sections with material away from the neutral axis are enclosed in an efficient region (light grey) bordered by limit curves $1 \mathrm{v}$ and $1 \mathrm{~h}$. In this region $1<p_{s}<3$. Cross-sectional shapes with material close to the neutral axis lie in an inefficient region (dark grey) delimited by curves $1 \mathrm{~h}$ and $2 \mathrm{v}$ where $0<p_{s}<1$. Similar to curves $1 \mathrm{v}, 2 \mathrm{v}$ and $1 \mathrm{p}$ plotted for rectangles, curves with equivalent meanings are plotted in Fig. 4 for hollow ellipses.

Fig. 4 shows that cross-sections satisfying the design requirements, $F^{\prime} /\left(E \times I_{D}\right)$, lie on a horizontal line. The performance, $p_{s}=\psi_{I} / \psi_{A}=\lambda$, is visualized on the chart as the slope of the line between the origin and the co-ordinates of a cross-section. The greater the slope, the lighter the crosssection. The I section $C_{3}$, for example, is more efficient than those represented by $C_{1}$ and $C_{2}$.

\subsection{Shape selection charts for scaled cross-sections}

When the design choice is among scaled cross-sections with prescribed material, then the performance index is given by Eq. (16), which rearranged in a logarithmic form is written as:

$\log \psi_{I}=\frac{1}{q} \log \psi_{A}+\frac{1}{q} \log \bar{p}_{s}$

Eq. (21) is a line plotted in a logarithmic chart $\left(\log \psi_{A}\right.$, $\log \psi_{I}$ ), where the gradient, $1 / q$, is governed by the scaling transformation, and the $y$-intercept, $1 / q \log \bar{p}_{s}$, describes the level of performance. Fig. 5 is the equivalent of Fig. 4 but displayed on a logarithmic scale. Thus, Eq. (21) can be used to plot parallel lines of iso-performance. For a given scaling, the higher the $y$-intercept, $1 / q \log \bar{p}_{s}$, the lighter the cross-section.

An iso-performance line permits the comparison of performance for different shapes subjected to a given scaling, i.e. $1 / q=$ constant. Examples of iso-performance lines are shown in Fig. 5. For horizontal scaling, the selection line is $q=1$, while for proportional scaling, the selection line is $q=1 / 2$. The line representing $q=1 / 3$ guides the selection among vertically scaled sections. For instance, consider a height constraint that imposes a vertical scaling on an elliptical and a rectangular cross-section. If the ellipse is a first trial shape, then the rectangle lies above the iso-performance line $q=1$ and thus results in being lighter. In contrast, a vertical scaling $(q=1 / 3)$ gives opposite results. However, a lighter lightweight potential can be obtained with hollow rectangles lying above the guideline $q=1 / 3$.

\subsection{Co-selection of material and shape for non-scaled cross- sections}

When the design prescribes that the envelope does not vary, then the only variables are $M$ and $S$. The performance is governed by the product of material and shape properties, $p=\lambda E / \rho$. $G_{D}$ is constant and the co-ordinates locating a cross-section on a chart are:

$C\left(\frac{m}{G_{D}}=M \times S, \frac{F^{\prime}}{G_{D}}=M \times S\right)$

Fig. 6 plots the shape transformers (Table 2) for steel $\left(\rho=7.8 \mathrm{Mg} / \mathrm{m}^{3}, E=200 \mathrm{GPa}\right)$ and carbon fibre reinforced plastic (CFRP) $\left(\rho=1.6 \mathrm{Mg} / \mathrm{m}^{3}, E=160 \mathrm{GPa}\right)$ on a chart

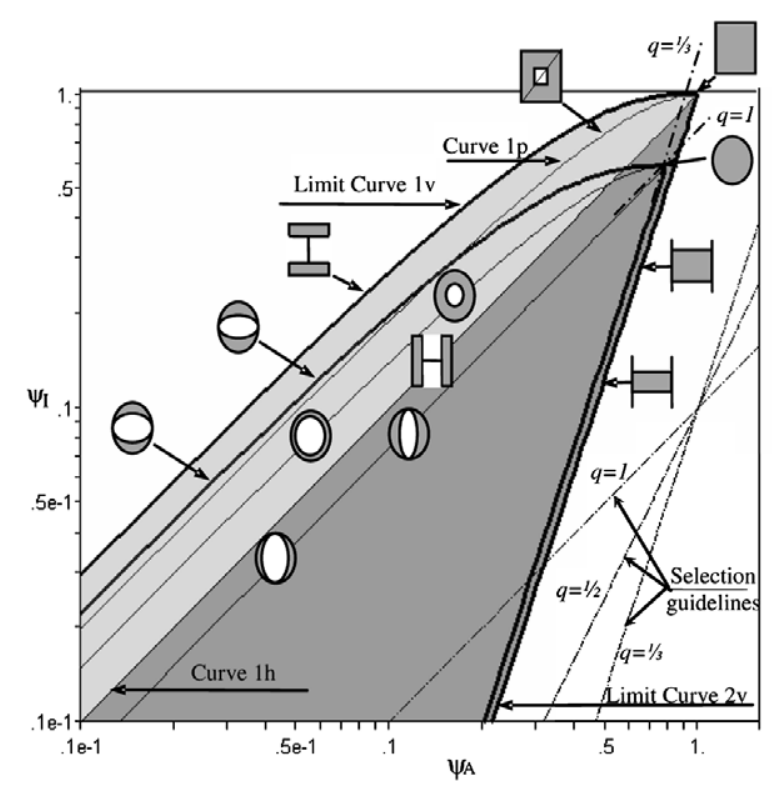

Fig. 5. Selection chart for cross-sections scaled horizontally $(q=1)$, vertically $(q=1 / 3)$, and proportionally $(q=1 / 2)$. Logarithmic scale. Legend: Light grey for efficient region, dark grey for inefficient region. 


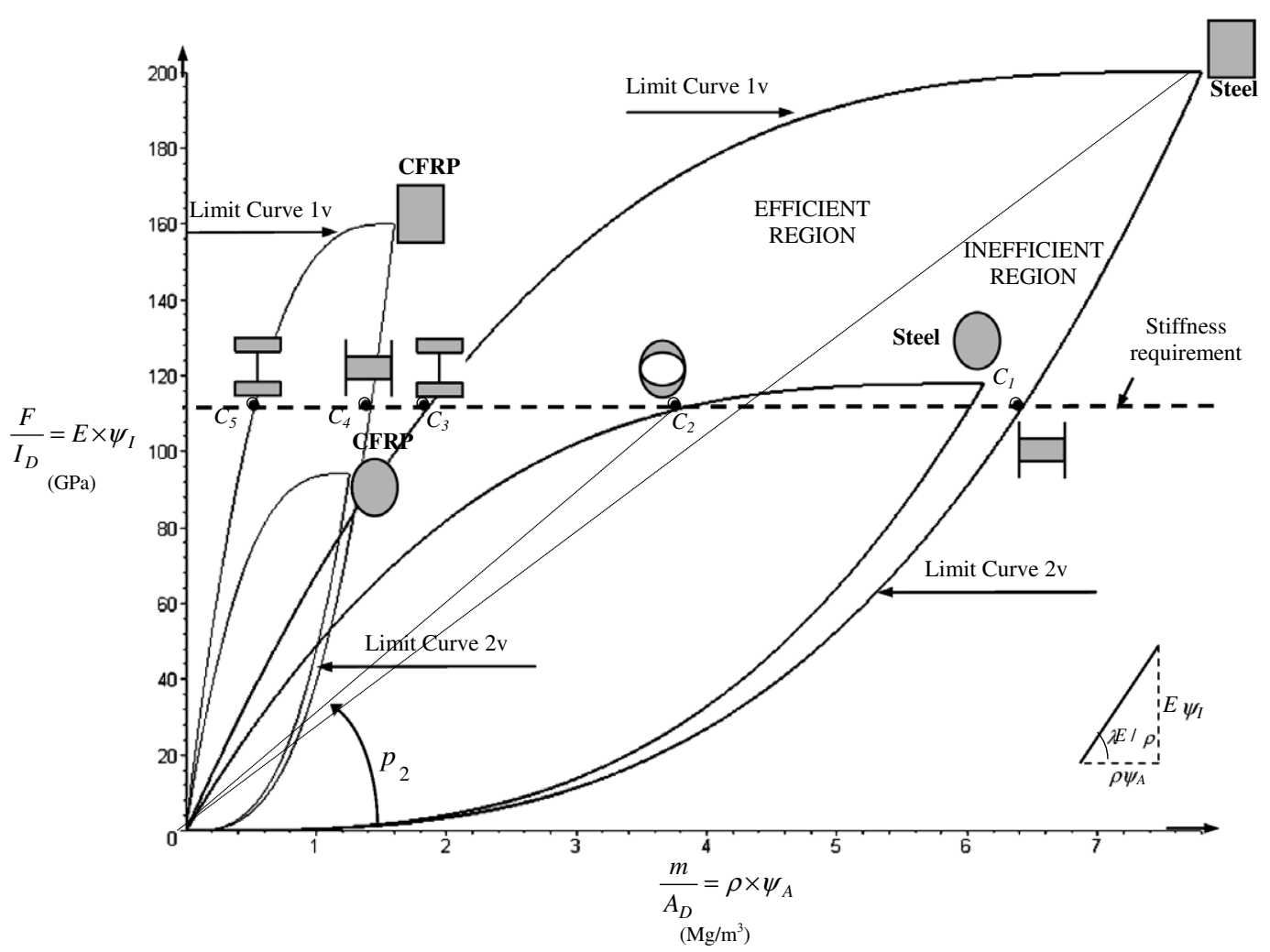

Fig. 6. Co-selection of shape and material for cross-sections not scaled.

$\left(\rho \psi_{A}, E \psi_{I}\right)$. It is found that for each material there are regions similar to those shown in Fig. 4. This means that CFRP and steel cannot be shaped into cross-sections whose properties lie outside the respective boundaries $1 \mathrm{v}$ and $2 \mathrm{v}$. Although curves $1 \mathrm{p}$ and $1 \mathrm{~h}$ can be plotted to give a broader spectrum of the shapes of a class, they have been omitted in Fig. 6 to simplify the reading.

Now, as an example, consider a stiffness requirement, such as that visualized in Fig. 6. The horizontal line crossing the shape boundaries for both materials implies that CFRP and steel are eligible to satisfy the functional requirements. Steel can be shaped with $\psi_{A}$ in the range $C_{1}-C_{3}$, while $\psi_{A}$ for CFRP can be between $C_{4}$ and $C_{5}$. Since the efficiency of a cross-section is visualized on the chart by the slope $p=\lambda E / \rho$, then even an optimum shaping of steel, such as cross-section $C_{3}$, cannot provide a beam as light as $C_{4}$, which is the heaviest beam made from CFRP.

\subsection{Co-selection of $M$ and $S$ for scaled cross-sections}

Envelope variation involves relative scaling between cross-sections. In this scenario, Eq. (16) drives the choice of the variables for a light and stiff beam. To visualize selection on logarithmic charts, we take the log of the performance index (16) and rearrange it as:

$\log E \psi_{I}=\frac{1}{q} \log \rho \psi_{A}+\frac{1}{q} \log \bar{p}$
Eq. (23) represents a line that defines points with the same performance. As seen in Section 4.3, such a line can be used to guide co-selection of material and shape for a given scaling. Examples of these lines are shown in Fig. 7, together with the plots of shape transformers (Table 2) for three materials: CFRP $\left(\rho=1.6 \mathrm{Mg} / \mathrm{m}^{3}, E=160\right.$ GPa), aluminium $\left(\rho=2.7 \mathrm{Mg} / \mathrm{m}^{3}, E=70 \mathrm{GPa}\right)$ and steel

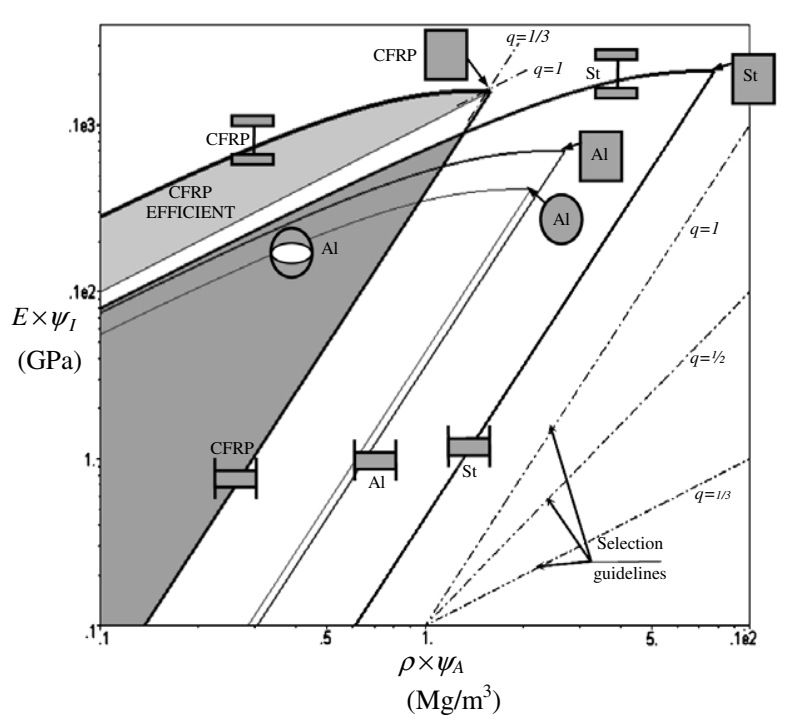

Fig. 7. Co-selection of shape and material is guided by iso-performance lines for a prescribed scaling (horizontal $q=1$, proportional $q=1 / 2$, vertical $q=1 / 3$ ). 
$\left(\rho=7.8 \mathrm{Mg} / \mathrm{m}^{3}, E=210 \mathrm{GPa}\right)$. Hollow rectangular crosssections are displayed for steel and CFRP, while curves of rectangular and elliptical classes of shapes are illustrated for aluminium.

A product of properties, $M \times S$, above a selection line identifies a cross-section lighter than those below. For example, consider in Fig. 7 a rectangular cross-section made from CFRP. For a horizontal scaling $(q=1)$, there are no lighter cross-sections of aluminium and steel. Only an efficient shaping of CFRP (light grey region) allows a performance improvement. However, if there is a width constraint $(q=1 / 3)$, then both steel and aluminium can be shaped more efficiently. Lighter solutions for steel and aluminium are enclosed in the dark grey region.

The brief examples given in Section 4 have shown that the use of shape transformers can be valuable to develop maps that widen the spectrum of choices for a light stiff beam. When materials are to be compared for different shapes classes, the shape transformers can ease the visual ranking and help to obtain insight into optimum selection.

\section{Concluding remarks}

This paper has introduced the methodology of shape transformers for efficiency modelling and graphical selection. Shape transformers are dimensionless measures of geometric quantities of a cross-section. They describe shape properties that do not change in value under the scaling of a cross-section. They enable the formulation of shape classes similarly to material classification.

Modelling with shape transformers allows the equations of continuous mechanics and the performance indices to be expressed in terms of design requirements, material, shape properties, and geometric quantities of the envelope. One advantage of this rationale lies in the relative ease in exploring how performance changes with different scaling conditions.

Performance indices derived for different design scenarios have been used to visualize iso-performance contours that guide the selection of beams in pure bending. This paper has shown that plotting the shape transformers on design charts improves the selection process and helps to obtain understanding about structural efficiency.

\section{References}

[1] Cox HL. The design of structures of least weight. Pergamon Press; 1965.

[2] Shanley FR. Weight-strength analysis of aircraft structures. 2nd ed. New York: Dover; 1960.

[3] Caldwell JB, Woodhead RG. Ship structures: some possibilities for improvement. North East Cost Inst - Inst Engrs Shipbuilders - Trans 1973;89:101-20.

[4] Parkhouse JG. Structuring a process of material dilution. In: Nooshin $\mathrm{H}$, editor. Proceedings of 3rd international conference on space structures. Elsevier Applied Science Publishers; 1984. p. 367-74.

[5] Ashby MF. Materials and shape. Acta Metall Mater 1991;39(6): 1025-39.

[6] Ashby MF. Material selection in mechanical design. 2nd ed. Oxford: Pergamon Press; 1999.

[7] Ashby MF, Bréchet YJM. Designing hybrid materials. Acta Mater 2003;51:5801-21.

[8] Pasini D. A new theory for modelling the mass-efficiency of material, shape and form. PhD thesis, Bristol University; 2003.

[9] Pasini D, Smith DJ, Burgess SC. Structural efficiency maps for beams subjected to bending. Proc Instn Mech Engrs Part L, J Mater: Design Appl 2003;217(3):207-20.

[10] Pasini D, Smith DJ, Burgess SC. Selection of arbitrarily scaled crosssections in bending stiffness design. Proc Instn Mech Engrs Part L, J Mater: Design Appl 2003;217(2):113-25.

[11] Pasini D, Burgess SC, Smith DJ. Performance indices for arbitrarily scaled rectangular cross-sections in bending stiffness design. Proc Instn Mech Engrs Part L, J Mater: Design Appl 2002;216:101-13. 